UDK 78(4-014)"14"

\author{
Bernhold Schmid
}

Musikhistorische Kommission, Bayerische Akademie der Wissenschaften, München Glasbenozgodovinska komisija Bavarske akademije znanosti, München

\title{
Aspekte der Überlieferung von Mensuralmusik in Zentraleuropa während des 15. Jahrhunderts
}

\section{Vidiki tradicije menzuralne glasbe v Srednji Evropi v 15. stoletju}

ZUSAMMENFASSUNG

Der Mensuralcodex St. Emmeram der Bayerischen Staatsbibliothek (Clm 14274) enthält zentraleuropäisches und auswärtiges Repertoire vom späten 14. Jahrhundert bis unmittelbar in Entstehungszeit der Handschrift (zwischen ca. 1436 und den frühen vierziger Jahren des 15. Jahrhunderts). Wie in keiner anderen Quelle spiegelt sich das Repertoire und die Überlieferungssituation von mehrstimmiger Musik vor der Mitte des 15. Jahrhunderts in Zentraleuropa; der Codex ist deshalb bestens geeignet, uns über das Repertoire, den Kenntnisstand und das Niveau einheimischer Musiker zu unterrichten. Dies ist selbstverständlich durch die Analyse zentraleuropäischer Musik und ihres Vergleichs mit auswärtigem Repertoire möglich. Im vorliegenden Beitrag wird jedoch versucht, durch das Studium zentraleuropäischer Überlieferung von fremdländischer Musik Auskunft über das Niveau der Musiker in Zentraleuropa zu erhalten. Dies geschieht zunächst anhand der beiden Aufzeichnungen von Binchois' Chanson "C'est assez pour morir" im Emmeramer Codex (Nr. 247, fol. 127r und Nr. 250, fol. 129r) im Vergleich mit der besten Quelle für dieses Stück, dem älteren der beiden Escorial-Codices E-E V.III.24 (fol. 34v-35r). Die untextierte Aufzeichnung Nr. 247 aus Emmeram entspricht hinsichtlich des Notentexts (von geringfügigen Abweichungen abgesehen) der Aufzeichnung im Codex Escorial. Dies zeigt, daß frankoflämische Kompositionen schon rasch nach ihrer Entstehung in vorzüglichen Abschriften im zentraleuropäischen Raum verfügbar waren - zwischen
POVZETEK

Menzuralni kodeks St. Emmeram v bavarski Staatsbibliothek (Clm 1427) vsebuje srednjeevropski in zunanji repertoar, ki sega od poznega 14. stoletja neposredno do časa nastanka rokopisa (med ok. 1436 in začetkom štiridesetih let 15 . stoletja). V nobenem drugem viru mimo tega se ne zrcalita repertoar in situacija izročila večglasne muzike pred sredino 15. stoletja v Srednji Evropi; kodeks je zato zelo primeren, da nas pouči o repertoarju, poznavanju in ravni domačih glasbenikov. Seveda, to je mogoče narediti $z$ analizo srednjeevropske glasbe in primerjavo le-te $z$ zunanjim repertoarjem. Avtor skuša $v$ prispevku prek analize repertorija iz tujih dežel v srednjeevropskem izročilu dobiti informacijo o ravni glasbenikov v Srednji Evropi. To se najprej zgodi ob obeh zapisih Binchoisove šansone "C'est assez pour morir" v kodeksu iz Emmerama (št.247, fol.127r in št. 250, fol. 129r) v primerjavi $\mathrm{z}$ najboljšim virom za to skladbo, starejšim od obeh Escorial kodeksov E-E V.III.24 (fol.34v-35r). Zapis brez besedila št. 247 iz Emmerama ustreza glede notnega zapisa ( $\mathrm{z}$ drobnimi odstopanji) zapisu $\mathrm{v}$ kodeksu Escorial. To kaže, da so bile frankoflamske kompozicije kmalu po nastanku dosegljive $\mathrm{v}$ odličnih prepisih $\mathrm{v}$ srednjeevropskem prostoru med kodeksom Escorial in zapisom "C'est assez" $\mathrm{v}$ Emmeramu je približno desetletje razlike. Št. 250 kodeksa Emmeram (kontrafakt "Virgo rosa venustatis" je podpisal pisec D) pa bistveno odstopa; odlomek je tako slab, da ga je treba označiti za "pokvarjenega". Pri natančnejši obravnavi se vendarle 
dem Codex Escorial und der Aufzeichnung des „C'est assez" in Emmeram dürften nur etwa 10 Jahre liegen - und erstklassig kopiert wurden. Nr.250 des Emmeramer Codex (ein von Schreiber D notiertes Kontrafakt "Virgo rosa venustatis") hingegen weicht verschiedentlich $\mathrm{ab}$; eine Stelle ist dermaßen schlecht überliefert, daß man sie als "verderbt" bezeichnen möchte. Bei näherem Betrachten ergibt sich indessen, daß die Änderungen als Korrekturversuche zu werten sind. Dem Schreiber mag eine schlechte Kopie vorgelegen haben, die er dann zu verbessern versuchte. Das Ergebnis ist bedeutend schlechter als Binchois' Original. Der Korrekturversuch zeigt jedoch, daß der Schreiber seine Vorlage als fehlerhaft erkannt hat, und daß er in der Lage war, korrigierend einzugreifen, wenn er auch das Original qualitativ nicht erreicht hat. Vorstellungen von frankoflämischer Satztechnik muß er also gehabt haben.

Häufig ist in zentraleuropäischen Quellen Dreistimmigkeit zur Zweistimmigkeit reduziert, indem der Contratenor weggelassen wird. Nicht selten sind dabei die beiden notierten Stimmen Cantus und Tenor in ihrer Faktur vereinfacht. Der erwähnte Schreiber D hat mehrmals fehlende Contratenores nachgetragen, so auch im Fall von Nr.274 des Codex Emmeram, einem Gloria von Hugo de Lantins (in Emmeram jedoch Forest zugeschrieben). Seine Nachträge von Contratenores zeigen ihn ebenfalls als einen Musiker, der den Versuch unternimmt, das Niveau frankoflämischer Komponisten zu erreichen, indem er deren Sätze in Originalgestalt überliefern will. Beim genannten Gloria mißlingt dies: Vergleicht man die Aufzeichnung in Emmeram durch den Schreiber D mit der konkordanten Überlieferung in I-Bc Q 15 (fol.84v-85r), so zeigt sich, daß in die Emmeramer Fassung zwei Quellen unterschiedlicher Qualität eingegangen sind: Der Contratenor ist unverändert überliefert, dem Außenstimmenpaar liegt jedoch eine rhythmisch vereinfachte zweistimmige Version zugrunde, was dem Schreiber D offenbart nicht aufgefallen ist. izkaže, da so spremembe poskusi korekture. Pisec je morda dobil v roke slabo kopijo, ki jo je skušal izboljšati. Rezultat je precej slabši od Binchoisovega izvirnika. Poskus korekture pa kaže, da je prepisovalec prepoznal predlog za pomanjkljivo in da se je bil sposoben lotiti korigiranja, četudi kakovostno ni dosegel izvirnika. Predstave o frankoflamski stavčni tehniki je torej moral poznati.

V srednjeevropskih virih je triglasje pogosto skrčeno na dvoglasje, tako da je izpuščen kontratenor. Neredko sta pri tem oba zapisana glasova, kantus in tenor, po svoji fakturi poenostavljena. Omenjeni pisec $\mathrm{D}$ je večkrat dodal manjkajoče kontratenorje, tudi v primeru št. 274 kodeksa Emmeram, Gloriji Huga de Lantinisa ( $v$ Emmeramu pripisano Forest). Njegovi pripisi kontratenorjev ga razkrivajo kot glasbenika, ki želi njihove stavke posredovati v izvirni obliki. Pri omenjeni Gloriji se mu to ni posrečilo. Primerjava zapisa v Emmeramu izpod peresa pisca D z ujemajočo se verzijo v I Bc Q 15 (fol.84v-85r) pokaže, da sta v inačici iz Emmerama prisotna dva vira različne kakovosti: kontratenor je prevzet brez sprememb, zunanjima glasovoma pa je za osnovo ritmično poenostavljena dvoglasna različica, ki piscu D očitno ni bila všeč.

Der Mensuralcodex St. Emmeram der Bayerischen Staatsbibliothek München (DMbs Clm 14274), eine Handschrift, die wie keine andere das Repertoire und die Überlieferungssituation von mehrstimmiger Musik im zentraleuropäischen Raum spiegelt, ${ }^{1}$

1 Die engen Beziehungen des Codex zu Wien - einige Teile dürften dort entstanden sein - faßt Reinhard Strohm, Native and Foreign Polyphony in Late Medieval Austria, in: MD 38, 1984, S.218f zusammen. Er vertritt die These, daß das Repertoire von D-Mbs Clm 14274 dem der Schule und Kapelle von St. Stephan entspricht. (Weitere Literatur zur Entstehung der Quelle etc. bei Strohm in Fußn.) 
enthält als Nr. 247 und als Nr. 250 Binchois' Chanson "C'est assez pour morir«. Das Stück ist somit zweimal nahezu benachbart innerhalb desselben Faszikels, jedoch von zwei verschiedenen Schreibern aufgezeichnet. Der von Ian Rumbold so genannte Schreiber D, ${ }^{2}$ neben dem Besitzer und Kompilator Johann Poetzlinger der zweite Hauptschreiber der Handschrift, ist für die Notierung von Nr. 250 verantwortlich: Er textiert den Satz mit "Virgo rosa venustatis", was der Tendenz der Quelle entspricht, weltliche Texte grundsätzlich zur geistlichen Kontrafaktur umzugestalten. Nr. 247 wurde von einem der in der Handschrift zahlreichen subsidiary scribes - wie Rumbold sie nennt - geschrieben: er gibt in allen drei Stimmen nur jeweils das originale Textincipit an, während seine Aufzeichnung ansonsten untextiert bleibt. Außer in DMbs Clm 14274 ist der Satz schließlich noch im älteren der beiden Escorial-Codices (E-E V.III.24) überliefert. ${ }^{3}$

Betrachtet man beide Aufzeichnungen aus D-Mbs Clm 14274 hinsichtlich des Notentexts näher und vergleicht man sie mit der Notierung in E-E V.III.24, so ergeben sich auch hier charakteristische Abweichungen:

Notenbeispiel 1a

D-Mbs 14274, Nr.250, M.1-4:

D-Mbs 14274, Nr.247, M.1-4:

E-E V.III.24, M.1-4:
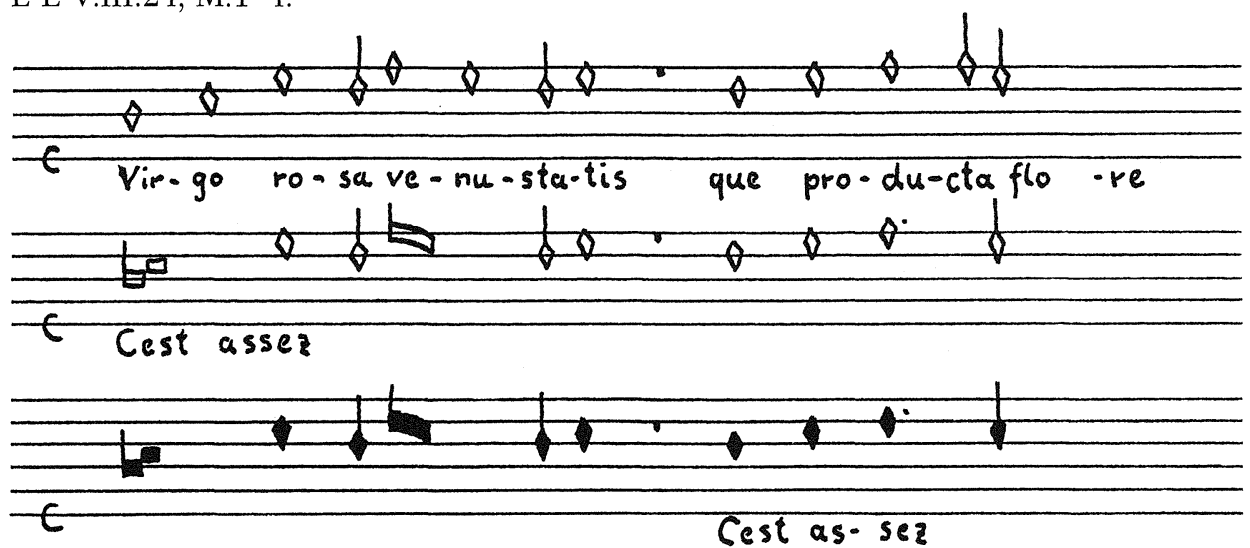

2 Vgl. Ian Rumbold, The Compilation and Ownership of the 'St Emmeram' Codex (Munich, Bayerische Staatsbibliothek, Clm 14274), in: Early Music History 2, Cambridge usw. 1982, S. 161-235.

3 D-Mbs Clm 14274 Nr. 247: fol.127r; Nr. 250: fol.129r. (Die Numerierung erfolgt nach Karl Dèzes, Der Mensuralcodex des Benediktinerklosters Sancti Emmerami zu Regensburg, in: Zeitschrift für Musikwissenschaft 10, 1927/28, S.65-105.) Ausgaben: Wolfgang Rehm, Die Chansons von Gilles Binchois, Mainz 1957, S.10. Das Kontrafakt ist ediert von A. Parris, The Sacred Works of Gilles Binchois, Diss. Bryn Mawr College 1965, Nr.63.

Escorial: E-E Ms. V.III.24, fol.34v-35r. Faksimileausgabe: Wolfgang Rehm (Hrsg.), Codex Escorial: Chansonnier, Kassel 1958. 
Notenbeispiel $1 \mathrm{~b}$

D-Mbs 14274, Nr.250, M.5-7,1:

D-Mbs 14274, Nr.247, M.5-7,1:

E-E V.III.24, M.5-7,1:

(Color ist durch hohle Noten angegeben)
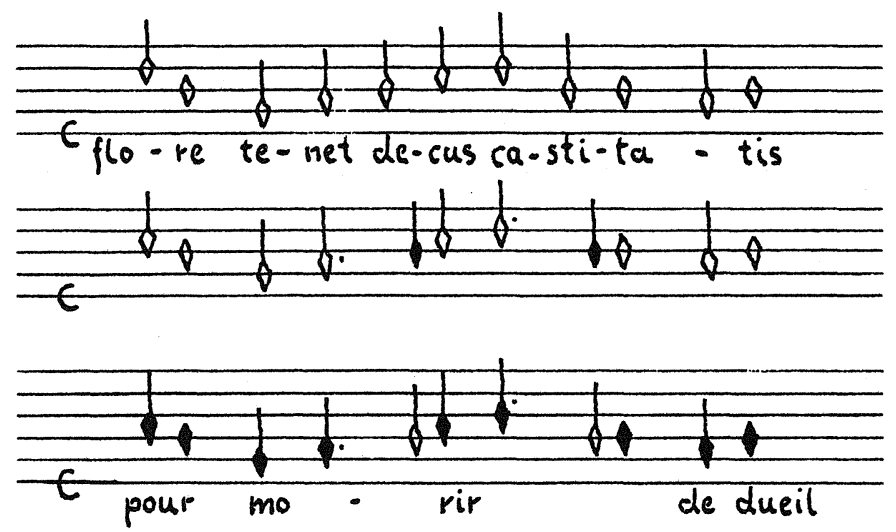

Notenbeispiel 1c

D-Mbs 14274, Nr.250, M.13,3-18,1:

D-Mbs 14274, Nr.247, M.13,3-18,1:

E-E V.III.24, M.13,3-18,1:

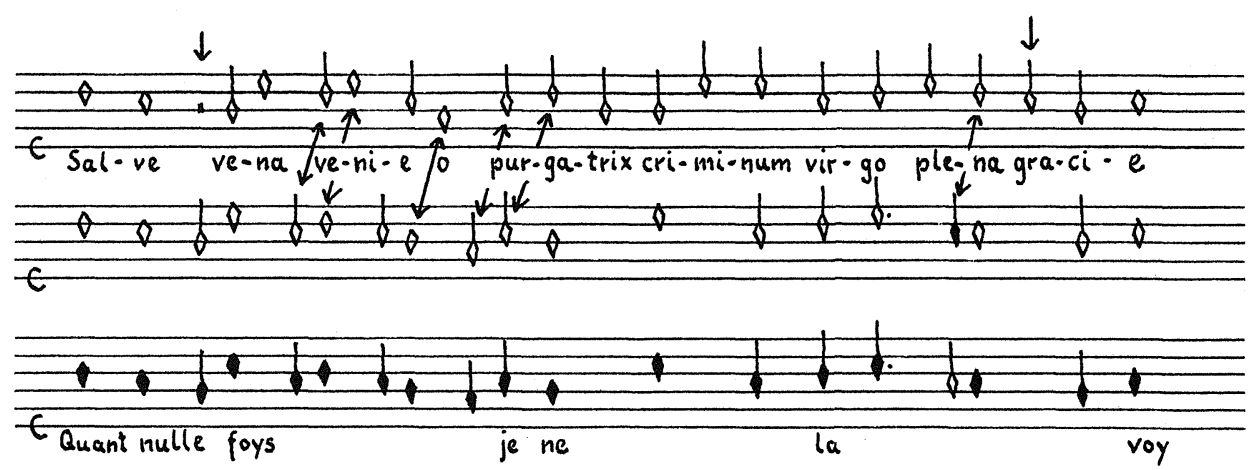

Bei allen gezeigten Beispielen jeweils aus der Oberstimme fällt auf, daß die Nr. 247 aus D-Mbs Clm 14274 und die Überlieferung in E-E V.III.24 große Ähnlichkeit haben, ${ }^{4}$ während die Notierung Nr. 250 aus Clm 14274 deutlich abweicht. Bei den

4 Dennis Slavin, Questions of Authority in Some Songs by Binchois, in: Journal of the Royal Musical Association 117/1, 1992, S.37, Fußn.37 behauptet, es gäbe zahlreiche Fehler und Varianten in D-Mbs Clm 14274. Im Fall von Nr.247 ist ihm zu widersprechen; der von ihm angeführte kritische Bericht von Rehms Ausgabe (vgl. Fußn.3) zeigt dies auch deutlich. 
ersten Beispielen werden aus Gründen der Unterlegung mit neuem, lateinischem Text längere Notenwerte oder Ligaturen aufgebrochen, ein selbstverständlich scheinender Vorgang, der jedoch nicht immer durchgeführt wird: im Emmeramer Codex lassen sich mehrere Fälle zeigen, bei denen die Notierung der textierten Stimme dem neuen Text nicht angepaßt wird. ${ }^{5}$ Sodann ist in der Aufzeichnung Nr.250 zu beobachten, daß Rhythmen vereinfacht werden: Punktierte Noten mit nachfolgendem kleineren Notenwert werden durch zwei identische Werte ersetzt. Schließlich ergeben sich größere Abweichungen vom ursprünglichen Notentext in der Oberstimme (und übrigens auch am Ende des Contratenors) von Nr.250: Bei "purgatrix criminum" werden wiederum längere Notenwerte in kürzere zerlegt, um den Text unterzubringen, und bei "virgo plena gracie" wird der Rhythmus vereinfacht wiedergegeben. Der wesentliche Unterschied ist jedoch folgender: In Nr.250 findet sich nach der 2. Note eine überzählige Minima-Pause, die zu einer Verschiebung der Oberstimme führt; dafür wird aus der Semibrevis a' kurz vor Ende des Beispielabschnitts in Nr.250 eine Minima, um die überflüssige Minimapause vom Anfang wieder einzusparen. Nach der überzähligen Minimapause finden sich außerdem insbesondere bei „venie o purgatrix" Abweichungen hinsichtlich der Diastematie (vgl. die Einzeichnungen im obigen Notenbeispiel 1c - das h' bei "(ple)-na" dürfte ein Schreibfehler sein). Man könnte die Version dieses Schreibers einfach als "verderbt" abtun, dies ist jedoch eine vereinfachte Sichtweise des Sachverhalts. Die Änderungen in der Tonhöhe lassen sich sinnvoll nur als Korrekturversuche deuten, die aufgrund der Verschiebung der Oberstimme notwendig wurden. Es ergeben sich zwar bei "venie" und bei "virgo plena" klangliche Härten, die in der originalen Version in Nr.247 und in E-E V.III.24 nicht zu beobachten sind. Die aufgrund der Verschiebung notwendigen Korrekturen der Tonhöhen in Nr.250 führen aber zu einem deutlich besseren Ergebnis, als es zu erzielen gewesen wäre, wenn keine Änderungen bei der Diastematie vorgenommen worden wären, wie die folgenden Beispiele zeigen.

\footnotetext{
5 Textunterlegungsprobleme gibt es insbesondere dann, wenn bestimmte Passagen mehrfach textiert sind, so bei den Modellsätzen des Magnificat Nr.262 in D-Mbs Clm 14274.

Beim "Tota pulchra es" aus derselben Handschrift (Nr.261) - wir haben es nicht mit einer Kontrafaktur zu tun - sind mehrmals perfekte Breven anstatt einer Folge Brevis imperfecta - Semibrevis notiert, so daß der Text eigentlich nicht unterzubringen ist; die "richtige" Textierung bzw. Abfolge der Notenwerte ist den Vergleichsquellen zu entnehmen (I-Bc Q 15 [olim 37], fol.238v-239r; GB-Ob Ms. Can. misc. 213, fol.42v; F-Pn nouv. acquis. fr. 4379, fol.65r; I-Bu Ms. 2216, fol.33v-34r. F-Sm 222, fol.16r ist verloren, da Coussemaker diesen Satz nicht abgeschrieben hat).

Einen merkwürdigen Fall haben wir am Ende von D-Mbs Clm 14274, Nr.71 ("Recordare virgo mater" mit Tropus "Ab hac familia") vor uns: Der Cantus firmus liegt durchgehend in der Oberstimme und wird erst am Schluß in den Tenor verlagert. Der Text liegt weiterhin in der Oberstimme, wo jedoch zwei Worte fehlen, da er vermutlich nicht ganz unterzubringen war
} 
MUZIKOLOŠKI ZBORNIK • MUSICOLOGICAL ANNUAL XL

Notenbeispiel $2 \mathrm{a}$

D-Mbs 14274, Nr.250, M.13,3-18,1 mit durch Schreiber D vorgenommenen Änderungen in der Diastematie:

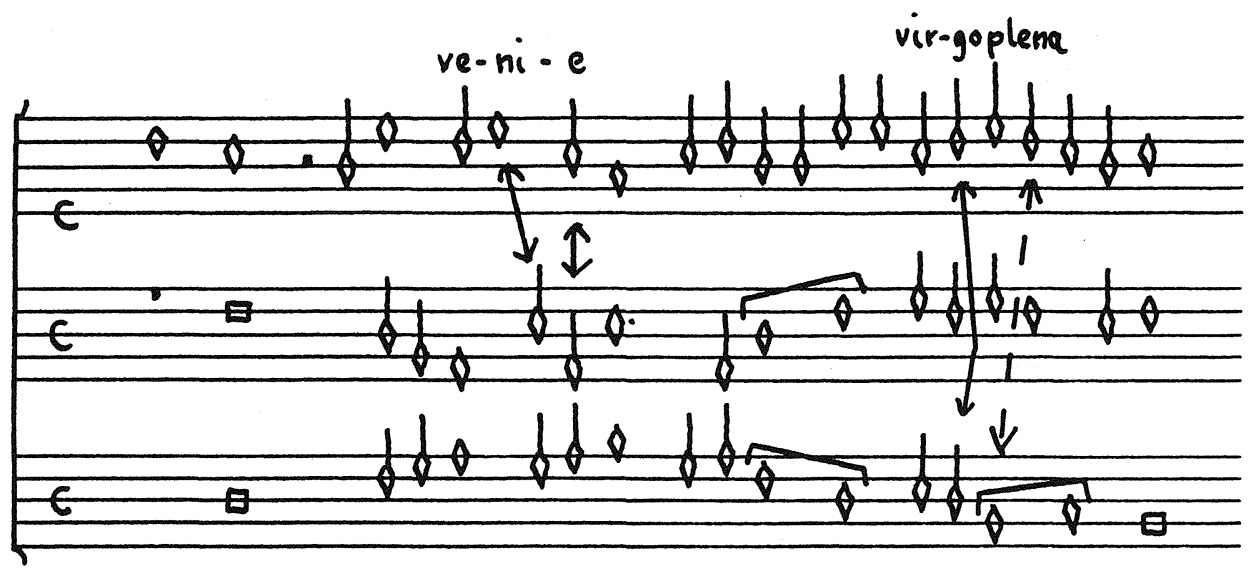

Notenbeispiel $2 \mathrm{~b}$

D-Mbs 14274, Nr.250, M.13,3-18,1 ohne Änderungen in der Diastematie:

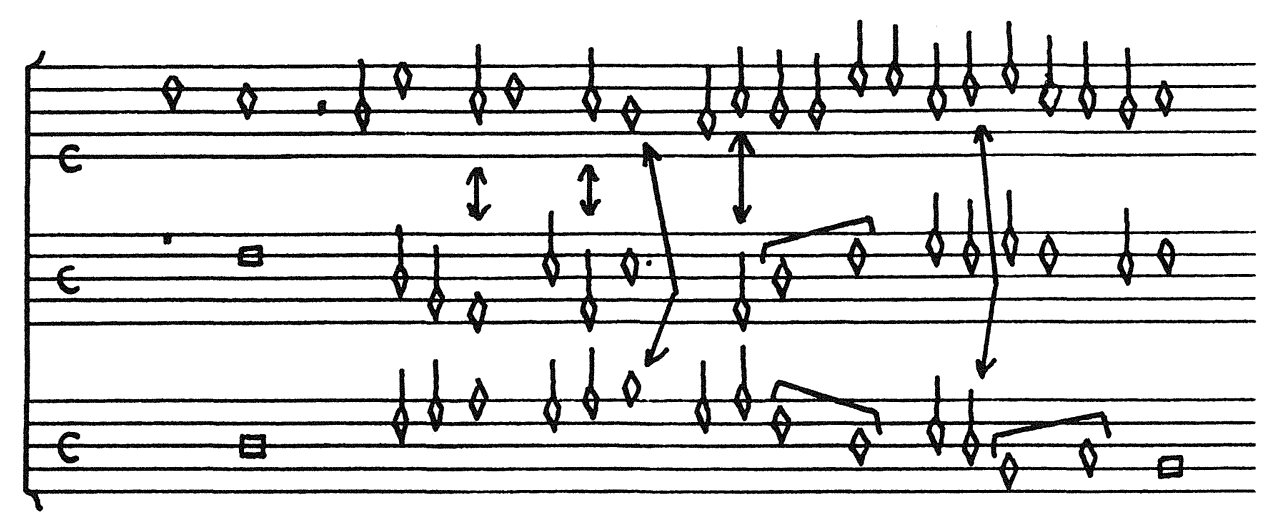


Notenbeispiel 2c

E-E V.III.24, M.13,3-18,1

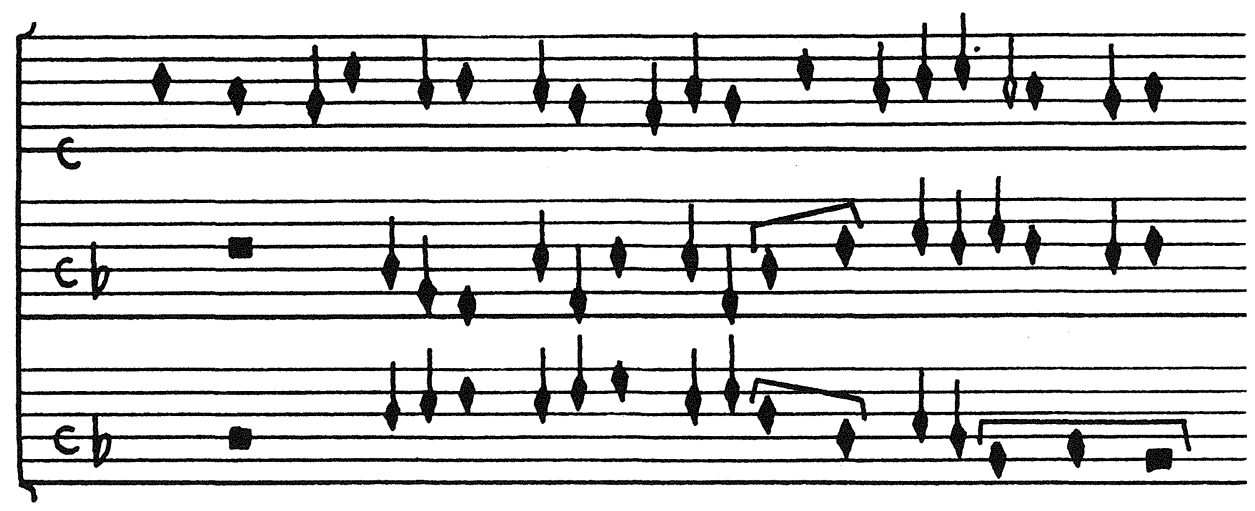

Korrigierende Eingriffe finden sich insbesondere beim Schreiber D häufiger. Im gezeigten Beispiel aus Nr.250 des Codex muß dem Schreiber für seine Abschrift eine fehlerhafte Quelle vorgelegen haben, die jene überflüssige Minimapause enthielt. Durch Auszählen der Notenwerte bis zur Kadenz stellte er fest, daß der Wert einer Minima überzählig war, worauf er vor der Kadenz den Wert einer Minima wegließ; dies ist ein bei Schreiber D und auch sonst im 15. Jahrhundert immer wieder zu beobachtendes Verfahren. ${ }^{6}$ Die Änderungen in der Diastematie mag er vorgenommen haben, indem er den Satz möglicherweise selbst zum Erklingen brachte und dabei korrigierende Eingriffe vornahm. Daß er möglicherweise die von ihm kopierten Sätze auch selbst aufgeführt hat, zeigt sich an der Tatsache, daß er vermutlich Organist gewesen ist: jedenfalls hat er in der Handschrift A-Wn 5094 einen Satz von Dufay zur Orgelpartitur umgeschrieben. ${ }^{7}$

Versuchen wir aus der Überlieferungssituation des "C'est assez" in D-Mbs Clm 14274 ein Fazit zu ziehen: Mit Nr. 247 haben wir eine Abschrift vor uns, die sehr nahe am zentralen Überlieferungsstrang liegt, wie die Nähe zu E-E V.III.24, einer zentralen Quelle für die Überlieferung der Chansons von Binchois, deutlich werden läßt. ${ }^{8}$ Es

${ }^{6}$ Vgl. Bernhold Schmid, Zur Edition des Mensural-Codex St. Emmeram der Bayerischen Staatsbibliothek München, in: Musik in Bayern 47, 1993, S.52-54 (Beispiele von Korrektur durch korrespondierendes Ergänzen oder Weglassen von Notenwerten ohne diastematische Veränderungen; es handelt sich ebenfalls um Schreiber D). Vgl. dazu generell Martin Just, Der Mensuralkodex Mus. ms. 40021 der Staatsbibliothek Preußischer Kulturbesitz Berlin, 2 Bände, Tutzing 1975, Bd.1, S.112.

7 Theodor Göllner, Notationsfragmente aus einer Organistenwerkstatt des 15. Jahrhunderts, in: Archiv für Musikwissenschaft 24, 1967, beschreibt S.172ff den Vorgang der Umschrift; Tom R. Ward, A Central European Repertory in Munich, Bayerische Staatsbibliothek, Clm 14274, in: Early Music History 1, Cambridge usw. 1981, S.342 klärt die Identität des Schreibers D aus D-Mbs Clm 14274 mit der des Schreibers der Orgelpartitur in A-Wn 5094; Bernhold Schmid, Notationseigenheiten Im Mensural-Codex St. Emmeram (Clm 14274) und Organistenpraxis, in: Musik in Bayern 43, 1991, S.52-68 beschreibt die teils eigenständige Notation des Schreibers D, die mutmaßlich aus seiner Tätigkeit als Organist zu erklären ist.

8 Vgl. Dennis Slavin, Art. Escorial-Handschriften, in: MGG (2.Aufl.) 3, Sp. 153-154: „Die von Schreiber 1 kopierten Lieder weisen auf eine enge Verbindung mit dem Komponisten selbst." "C'est assez" fol.34v-35r fällt unter die von Schreiber 1 notierten Sätze. 
zeigt sich dabei zum einen, wie rasch sich das neue frankoflämische Repertoire im deutschen Sprachraum verbreitet, da zwischen E-E V.III.24 und der Aufzeichnung von Nr.247 bzw. 250 in D-Mbs Clm 14274 nur etwa 10 Jahre liegen dürften. ${ }^{9}$ Zum anderen ist zu konstatieren, daß das Repertoire offensichtlich in qualitätvollen Abschriften kursierte. Wenn nur jeweils das Textincipit angegeben ist, dann mag man die Abschrift vielleicht als Grundlage für eine Umtextierung gesehen haben. Daß das denkbar ist, zeigt das vom selben Schreiber notierte "Mort en merchy"; ebenfalls eine Chanson von Binchois: über der Diskantstimme steht das Textincipit, darunter steht "Magnificat sexti toni ad omnes versus", ohne daß eine Textunterlegung vorgenommen worden wäre. Der Schreiber geht also einen Schritt weiter als beim "C'est assez", da festgelegt und angegeben wird, womit das Stück neu zu textieren ist. ${ }^{10}$

Mit Nr. 250 hingegen liegt eine Aufzeichnung vor, die zeigt, daß einige Schreiber in der Lage sind, fehlerhafte Vorlagen einigermaßen zu korrigieren. Das bedeutet, daß sie Vorstellungen von der Kompositionsweise, vom Satz frankoflämischer Meister haben und nicht nur kopieren.

Es sei zum Vergleich eine Passage aus einem anderen in D-Mbs Clm 14274 überlieferten Satz vorgeführt, nämlich die Textstelle "propter magnam gloriam tuam" aus einem Gloria, das als Nr.274 (fol.152v-153r) in unserer Handschrift enthalten ist, außerdem in der Handschrift I-Bc Q 15 (Nr.86, fol.84v-85r) und in I-TRmd 93 (fol.172v173r) sowie davon abhängig in I-TRmn 90 (fol.142v-143r). In Clm 14274 wird das Stück Forest zugeschrieben, in Bc Q 15 hingegen Hugo de Lantins. Aufgrund der zuverlässigeren Zuschreibungen in der italienischen Quelle, außerdem weil englische Merkmale im Stück nicht zu finden sind, dürfte es sich um ein Stück von Hugo de Lantins handeln. Schreiber in Clm 14274 ist wie bei Nr.250 der von Rumbold so genannte Schreiber D.

\footnotetext{
9 Slavin datiert E-E V.III.24 in die 1430er Jahre (vgl. seinen Art. Escorial-Handschriften, in: MGG 3, Sp.152), die weiß geschriebenen Faszikel von D-Mbs Clm 14274 dürften Rumbold, Compilation, S.206/207 zufolge aus den frühen 1440er Jahren stammen (vgl. auch Reinhard Strohm, Zur Datierung des Codex St. Emmeram (Clm 14274): Ein Zwischenbericht, in: Quellenstudien zur Musik der Renaissance II: Datierung und Filiation von Musikhandschriften der Josquin-Zeit, Wiesbaden 1983, S.235 kommt zu einem ähnlichen Ergebnis. Dagmar Braunschweig-Pauli, Studien zum sogenannten Codex St. Emmeram. Entstehung, Datierung und Besitzer der Handschrift München, Bayerische Staatsbibliothek, Clm 14274 (Olim Mus. ms. 3232a), in: Kirchenmusikalisches Jahrbuch 66, 1982, S.46, datiert hingegen denjenigen Abschnitt der Handschrift, der Nr.250 enthält, in die Jahre zwischen 1456 und 1459). - Die Beobachtung, daß zwischen der zentralen Tradition für Binchois' Chanson und der Überlieferung in D-Mbs Clm 14274 kein allzu großer Zeitraum liegen dürfte, ist keineswegs singulär: vgl. Lorenz Welker, New light on Oswald von Wolkenstein: central European traditions and Burgundian polyphony, in: Early Music History 7, Cambridge usw. 1987, S.191/192, wo er dasselbe Phänomen für Oswald von Wolkensteins Kontrafakturen feststellt.

${ }^{10}$ D-Mbs Clm 14274, Nr.245, fol.126v. Vgl. aber Dennis Slavin, Genre, final and range: unique sorting procedures in a fifteenth-century chansonnier, in: Musica Disciplina 43, 1989, S.115, Fußn.3, der unter Berufung auf David Fallows' Art. Binchois in New Grove Dictionary of Music and Musicians anzweifelt, daß es sich tatsächlich um das Kontrafakt einer Chanson handelt.
} 
Notenbeispiel 3a

D-Mbs 14274, Nr.274, M.17-24:

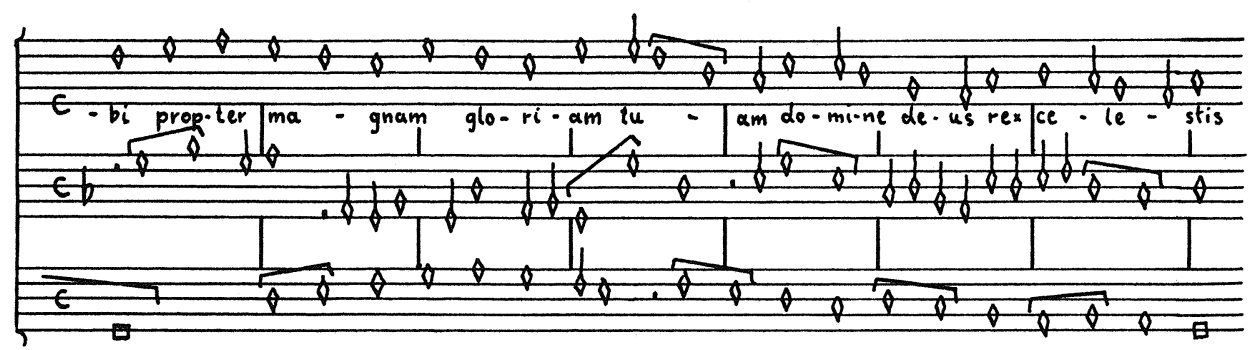

Notenbeispiel $3 \mathrm{~b}$

I-Bc Q 15, M.17-24:

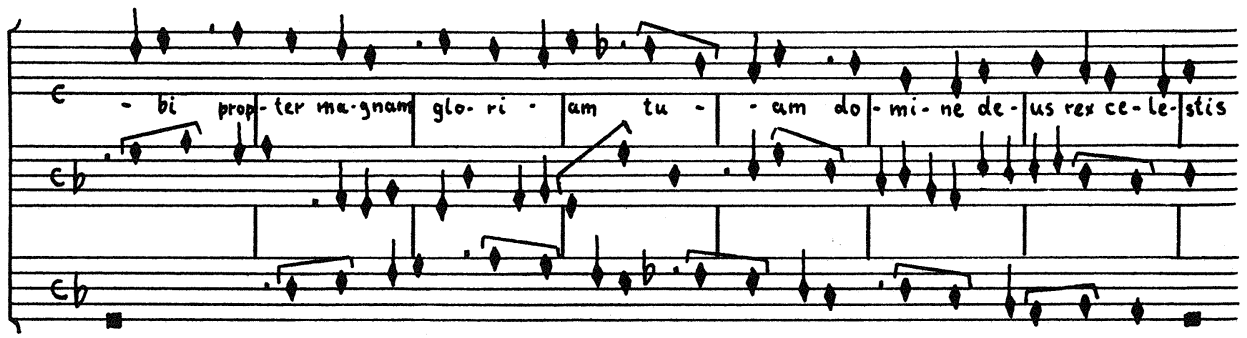

Die Gegenüberstellung der Aufzeichnungen aus I-Bc Q 15 - I-TRmd 93 und TRmn 90 entsprechen Bc Q 15 - und Clm 14274 zeigt Varianten beim rhythmischen Gefüge der Außenstimmen. ${ }^{11}$ Die Konzeption beider in Bc Q 15 stark konstruktiv gestalteten Stimmen wird in Mbs Clm 14274 durch einen sehr viel schlichteren Satz ersetzt, der zunächst Note gegen Note verläuft. Identisch hingegen ist in allen Quellen der Contratenor. In der Fassung aus Bc Q 15 und TRmd/TRmn gibt es keinerlei Probleme bei der Dissonanzbehandlung, während die in den Außenstimmen vereinfachte Version aus Mbs Clm 14274 mitunter erhebliche Dissonanzen aufweist. ${ }^{12}$ Wieder ist zu fragen, ob wir es mit einer verderbten Aufzeichnung im eigentlichen Sinn zu tun haben. Betrachten wir das Cantus-Tenor-Paar in Mbs Clm 14274, so ergeben sich nämlich auch hier keine größeren Probleme, ${ }^{13}$ so daß also der unverändert übernommene Contratenor dissoniert. In dieser Weise zweistimmig betrachtet ergibt der Satz zweifellos mehr Sinn als mit dem Contratenor. Einfach von einer fehlerhaften Aufzeichnung auszugehen wäre verfehlt. Es sei ein Erklärungsversuch gemacht: In deutschen

\footnotetext{
${ }^{11}$ Die Minimapause im Tenor bei "tu-(am)" fehlt im Manuskript. Fügt man sie ein, dann entspricht die Passage der Lesart von $\mathrm{Bc} \mathrm{Q} 15$.

12 Z.B. bei: "(ma)-gnam": f e'; "glo-(riam)«: c' h'; "(glo)-ri-(am)«: g f' a'; "(glori)-am": f e' etc.

${ }^{13}$ Hinzuweisen ist lediglich auf die Quarte bei "-gnam".
} 
Quellen finden sich häufig zur Zweistimmigkeit reduzierte und auch sonst vereinfachte Überlieferungen von ursprünglich dreistimmigen Sätzen. ${ }^{14}$ Eine derart vereinfachte Aufzeichnung könnte die Vorlage für die Fassung in Mbs Clm 14274 gewesen sein. Nun hat aber gerade der Schreiber D in dieser Quelle gelegentlich die Contratenores nachgetragen. ${ }^{15}$ Möglicherweise ist seine Notierung deshalb aus zwei verschiedenen Quellen zusammengeflossen: ursprünglich mag ihm der Satz in zweistimmiger Fassung vorgelegen haben, nachträglich wurde ihm eine Quelle mit Contratenor bekannt, er trug deshalb diese dritte Stimme mit ein. Die sich ergebenden klanglichen Probleme sofort zu erkennen macht die Stimmnotation unmöglich. In einer Aufzeichnung des Satzes treffen somit zwei Überlieferungen aufeinander, die verschiedene Entwicklungsstufen repräsentieren. Es zeigt sich die Heterogenität der Überlieferung so extrem wie selten anhand einer einzelnen Aufzeichnung.

Wie sind die Detailergebnisse der obigen Vergleiche verschiedener Aufzeichnungen eines Stücks zu werten? Welche Schlüsse sind daraus zu ziehen, bzw. wie lassen sich unsere Beobachtungen in einen größeren musikgeschichtlichen Zusammenhang einordnen? Wie Mbs Clm 14274 deutlich macht, ist das Repertoire im zentraleuropäischen Raum weit gestreut: ${ }^{16}$ retrospektive einheimische Mehrstimmigkeit steht neben französischem, englischem und italienischem Repertoire aus dem späten 14. Jahrhundert. Daneben sind moderne frankoflämische Sätze in verschiedensten Überlieferungsstadien enthalten: mehrere Sätze werden durch Weglassen des Contratenors zur Zweistimmigkeit reduziert und damit vielleicht den eigenen, evtl. bescheideneren Vorstellungen von Mehrstimmigkeit angeglichen. Andere Kompositionen weichen in Mbs Clm 14274 mehr oder weniger stark von sogenannten "guten" Quellen ab - ein Beispiel dafür ist die besprochene Version des "C'est assez" von Schreiber D - während die Aufzeichnung diverser Sätze in Clm 14274 von "guten" Handschriften kaum zu unterscheiden ist (vgl. die ebenfalls besprochene Nr.247). Schließlich enthält die Handschrift eine Anzahl von Kompositionen des Wiener Kantors Hermann Edlerauer, von Blasius, Kungsperger etc. als Unica, sowie ein auch sonst verbreitetes "Kyrie fons bonitatis" von Petrus Wilhelmi, ${ }^{17}$ die nach Art moderner frankoflämischer Chansonsätze gestaltet sind. Mögen sie auch deren Geschmeidigkeit nicht erreichen, ${ }^{18}$ so ist doch eindeutig, daß sich frankoflämische Satztechniken auch in Zentraleuropa auszubreiten beginnen, ${ }^{19}$ daß man sich jedenfalls in einem Adaptions- und Lernprozeß befindet, daß man dabei ist, Rückstände aufzuholen.

Soweit zur Repertoirestreuung in D-Mbs Clm 14274, die die stilistische Breite der im zentraleuropäischen Raum vorhandenen Musik eindrucksvoll repräsentiert. Der

\footnotetext{
${ }^{14}$ In Mbs Clm 14274 sind das z.B. folgende Sätze: Nr.31, 78, 102, 117, 134, 157, 172, 195, 236.

${ }^{15}$ Z.B. bei Nr.14/16 und 192; die Nr.54 ist der Contratenor zu Nr. 51, wie Lorenz Welker festgestellt hat (vgl. Lorenz Welker, Dufay songs in German manuscripts, in: John Kmetz (Hrsg.), Music in the German Renaissance. Sources, Styles, and Contexts, Cambridge 1994, S.11, Fußn.26.

${ }^{16}$ Zum Repertoire in Österreich generell vgl. Strohm, Polyphony, S.205ff.

${ }^{17}$ Wilhelmis "Kyrie fons bonitatisu findet sich in den I-TRmd 93, fol.94v-95r u. fol.358r-395r, sowie TRmn 90, fol.65v-66r.

${ }^{18}$ Dèzes, Mensuralcodex, S.72ff versucht einige Merkmale von Kompositionen Wilhelmis oder Edlerauers herauszuarbeiten, betrachtet diese jedoch nur vor dem Hintergrund von Dufay und Binchois und kommt deshalb zu einer ausschließlich negativen Sichtweise.

${ }^{19}$ Vgl. Strohm, Polyphony, S.225.
} 
MUZIKOLOŠKI ZBORNIK • MUSICOLOGICAL ANNUAL XL

Entwicklungsstand bzw. das Aufholen der in diesen Regionen entstandenen Kompositionen gegenüber den Satztechniken und der stilistischen Entwicklung insbesondere frankoflämischer Komponisten läßt sich nun zwar an einheimischen Sätzen ablesen. In meinem Beitrag ging es mir jedoch darum, zu zeigen, daß auch die Überlieferungssituation ausländischer Sätze in zentraleuropäischen Quellen den jeweiligen Entwicklungsstand sowie die Lern- und Adaptionsprozesse dokumentieren und beleuchten kann. So zeigt die Reduktion dreistimmiger Vorlagen zur Zweistimmigkeit bei gleichzeitiger rhythmischer Vereinfachung, wie sie in den Außenstimmen des besprochenen "Et in terra" (Mbs Clm 14274, Nr.274) zu beobachten ist, immerhin soweit die Fähigkeit des Umgangs mit Kompositionen von ausgereifter Satztechnik, als man in der Lage ist, die Vorlage umzuarbeiten, um das Stück dem eigenen bescheideneren Niveau anzupassen. Das häufig zu beobachtende Nachtragen von Contratenores läßt das erwachende Interesse an Originalfassungen erkennen: es wird nicht mehr als notwendig empfunden, die Sätze in vereinfachter Form zu übernehmen. Die Möglichkeit, korrigierende Eingriffe in fehlerhafte Vorlagen vorzunehmen, zeigt schließlich Kenntnisse oder wenigstens Vorstellungen von moderner Satztechnik. Am Ende derartiger an der Überlieferung ausländischer Musik abzulesender Lernvorgänge stehen Komponisten wie der Wiener Kantor Hermann Edlerauer etc. Besonders der für D-Mbs Clm 14274 so wichtige Schreiber D repräsentiert eine wesentliche Entwicklungsstufe auf diesem Weg, da er in der Lage ist, nicht nur abzuschreiben, sondern auch einzugreifen. Er korrigiert, ${ }^{20}$ trägt Contratenores teils nur nach, übernimmt sie teils aber auch unmittelbar - im Gegensatz zu diversen anderen Schreibern in Clm 14274 hat er jedenfalls keine Sätze in reduzierter Form notiert, sondern stets in vollständiger Dreistimmigkeit - er führt mutmaßlich Material aus verschiedenen Quellen zusammen, was seine Aufzeichnung des Glorias Nr. 274 wahrscheinlich macht, schließlich ist seine in A-Wn 5094 vermutlich für das Orgelspiel bestimmte Partitur einer Dufay-Chanson zu nennen. ${ }^{21}$ Somit dokumentiert er ein fortgeschrittenes Stadium beim Adaptionsprozeß frankoflämischer Satztechniken. Jedenfalls haben wir in ihm einen Musiker, nicht nur einen Kopisten vor uns. Möglicherweise ist er einer der "modernen" Komponisten wie Edlerauer, die mit Unica in Mbs Clm 14274 vertreten sind; er entstammt vielleicht unmittelbar dem Wiener Umkreis Edlerauers. ${ }^{22}$

\footnotetext{
${ }^{20}$ Gelegentlich korrigiert er auch von anderen Schreibern in Mbs Clm 14274 fehlerhaft aufgezeichnete Sätze: dies ist z.B. der Fall im "Ave tota casta" (Nr.192, fol.92v-93r), vgl. Schmid, Edition, S.50-51.

${ }^{21}$ Vgl. oben Fußn.7.

${ }^{22}$ Den Schreiber mit Edlerauer selbst zu identifizieren, dürfte abwegig sein, da Scribe D den Namen Edlerauer in durchaus verschiedener Weise schreibt.
} 